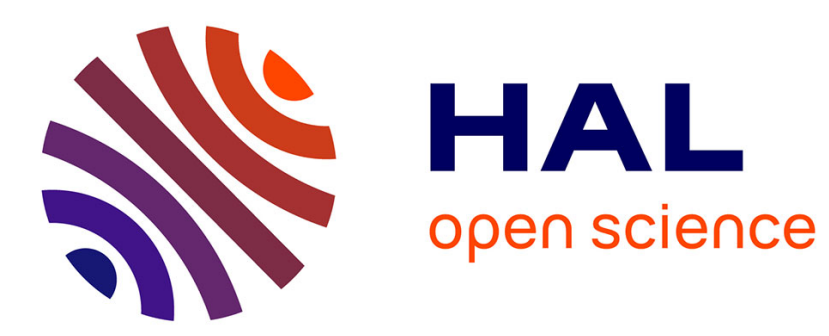

\title{
Control of Linear Min-plus Systems under Temporal Constraints
}

\author{
Saïd Amari, Isabel Demongodin, Jean Jacques Loiseau
}

\section{To cite this version:}

Saïd Amari, Isabel Demongodin, Jean Jacques Loiseau. Control of Linear Min-plus Systems under Temporal Constraints. 44th IEEE Conference on Decision and Control and European Control Conference (CDC-ECC 2005), Dec 2005, Seville, Spain. pp.7738-7743. hal-00362920

\section{HAL Id: hal-00362920 https://hal.science/hal-00362920}

Submitted on 19 Feb 2009

HAL is a multi-disciplinary open access archive for the deposit and dissemination of scientific research documents, whether they are published or not. The documents may come from teaching and research institutions in France or abroad, or from public or private research centers.
L'archive ouverte pluridisciplinaire HAL, est destinée au dépôt et à la diffusion de documents scientifiques de niveau recherche, publiés ou non, émanant des établissements d'enseignement et de recherche français ou étrangers, des laboratoires publics ou privés. 


\title{
Control of Linear Min-plus Systems under Temporal Constraints
}

\author{
S. Amari, I. Demongodin, Member, IEEE, and J.J. Loiseau
}

\begin{abstract}
We consider a class of controlled timed event graphs subject to strict temporal constraints. Such a graph is deterministic, in the sense that its behaviour only depends on the initial marking and on the control that is applied. This behaviour can be modelled by a system of difference equations that are linear in the Min-Plus algebra. The temporal constraint is represented by an inequality that is also linear in the min-plus algebra. Then, a method for the synthesis of a control law ensuring the respect of constraints is described. We give explicit formulas characterizing a control law, which, if two conditions are satisfied, ensures the validity of the temporal constraints. It is a causal state feedback, involving delays. The method is illustrated on an example.
\end{abstract}

\section{INTRODUCTION}

$\mathrm{W}$ E consider in the sequel a class of deterministic controlled processes subject to strict time constraints. Such time critical systems are frequent in the industry, for instance in the case of a thermal of chemical treatment, and also in the food industry. Of course the question is to validate some temporal conditions (see for instance $[1,5,6,7,8]$ ). In the present contribution, we formulate this problem in terms of a control problem, assuming that the process is subject to some control (it is generally the case) and proposing a method to solve the inverse problem, synthesizing of a control so that the constraint is validated. Following $[2,10]$, we shall use the formalism of timed event graph, and their algebraic models which are linear models over dioids [4]. Then the problem is formulated in terms of equations and of inequalities in the so-called Min-Plus algebra. The equation is the usual model representing the behaviour of a timed event graph, and the inequalities represent the temporal constraints to be validated. The control we are interested in is quite different from that considered within the so-called supervisory control framework [9, 11]. Here the time is explicitly taken into account At the contrary, the authors of $[2,10]$ consider timed event graphs and have used the formalism of dioids, but they treated other versions of such a problem. The first aim of their synthesis was rather a certain closed-loop property, expressed in terms of disturbance rejection or in terms of model matching, respectively, and the

S. Amari is with Institute of Research in Communications and Cybernetic of Nantes, 1 rue de la Noë, BP 92101, 44321 Nantes cedex 03, France (phone: +33-240376978 ; e-mail: said.amari@irccyn.ec-nantes.fr).

I. Demongodin is with Laboratory of Engineering for Automated Systems, 62 avenue Notre Dame du Lac, 49000 Angers, France (e-mail: isabel.demongodin@univ-angers.fr).

J.J. Loiseau is with Institute of Research in Communications and Cybernetic of Nantes, 1 rue de la Noë, BP 92101, 44321 Nantes cedex 03, France (phone: +33-240376967; e-mail: jean-jacques.loiseau@irccyn.ecnantes.fr). temporal constraint appeared as an additional requirement. We focus here on the validation of the time constraints, and the results are not easily comparable. Our work also differs from the existing literature on the control of (timed) discrete event systems, since the control laws we consider may involve some delays. In the present paper, we propose a method for the synthesis of a control that permits to validate a given set of time constraints. The control law itself is finally defined by a Min-Plus linear difference equation, involving a finite number of delays. Such an equation corresponds to a timed event graph, too. A first approach towards this method of synthesis was presented in [3]. The present contribution is a generalization for the timed event graphs subjected to several time constraints, and multiple controls.

The paper is organized as follows. In Section 2, some backgrounds are recalled, notably some notations concerning the Min-Plus semiring, the timed event graphs and their MinPlus linear models, the concept of a state equation. The problem, of finding a causal control verifying critical time constraints, is formulated in Section 3, and we propose in Section 4 a procedure for the control synthesis. We first consider the case of a single temporal constraint. Two conditions are proposed, which are sufficient for ensuring the existence of a solution. A simpler condition, which is satisfied in many practical cases, and is simpler to check, is also provided. Then we extend the method to the case of many different constraints. Section 5 is devoted to some illustrative examples, and finally Section 6 is devoted to the conclusion.

\section{BACKGROUNDS}

\section{A. Dioid algebra}

A monoid is a set, say $D$, endowed with an internal law, noted $\oplus$, which is associative and has a neutral element, denoted $\varepsilon, \forall a \in D, a \oplus \varepsilon=\varepsilon \oplus a=a$. A semiring is a commutative monoid endowed with a second internal law, denoted $\otimes$, which is associative, distributive with respect to the first law $\oplus$, has a neutral element, denoted $e$, and admits $\varepsilon$ like absorbing element: $\forall a \in D, a \otimes \varepsilon=\varepsilon \otimes a=\varepsilon$.

Finally, a diod is a semiring with an idempotent addition: $\forall a \in D, a \oplus a=a$. The dioid is called commutative if the second law $\otimes$ is commutative.

We shall consider in the sequel the so-called Min-Plus algebra that is $(\mathbb{R} \cup\{-\infty\} \cup\{+\infty\}$, min, +$)$. The Min-Plus 
algebra, denoted $\overline{\mathbb{R}}_{\text {min }}$ is a commutative dioid for which the law $\oplus$ is the operation min, having the neutral element $\varepsilon=+\infty$, and the second law, $\otimes$, is the usual addition, with neutral element $e=0$.

If $n \in \mathbb{N}$ and $v, w \in \overline{\mathbb{R}}_{\text {min }}^{n}$, we denote $v \oplus w$, the vector with components $v_{i} \oplus w_{i}:=\min \left(v_{i}, w_{i}\right)$ for $i=1$ to $n$. If no confusion can arise, when $p, q \in \mathbb{N}, A \in \overline{\mathbb{R}}_{\text {min }}^{p \times n}$ and $B \in \overline{\mathbb{R}}_{\min }^{n \times q}$ are given matrices, $A . B$ (or just $A B$ ) will denote the matrix multiplication in $\overline{\mathbb{R}}_{\min }$, defined by $(A . B)_{i j}=\bigoplus_{k=1}^{n}\left(A_{i k} \otimes B_{k j}\right):=\min _{k}\left(A_{i k}+B_{k j}\right)$

The Kleene star of a square matrix $M \in \overline{\mathbb{R}}_{\text {min }}^{n \times n}$, denoted $M^{*}$ is defined by $M^{*}=\bigoplus_{i \in \mathbb{N}} M^{i}$, where $M^{0}$ denotes the unit matrix, which entries equal $e$ on the diagonal, and $\varepsilon$ elsewhere. Let us recall that $v \in \overline{\mathbb{R}}_{\text {min }}^{n}$ then $x=M^{*} v$ is the maximal solution of both the inequality, $x \leq M . x \oplus v$, and the equality, $x=M \cdot x \oplus v[4]$.

\section{B. Timed event graphs and Min-Plus linear models}

An event graph is an ordinary Petri net where each place has exactly one upstream transition and one downstream transition. A timed event graph is obtained by associating delays to the places of an event graph. We shall use the following notations $P$ will denote the set of places of the considered graph, and $T$ its set of transitions. The number of transitions having at least one upstream place is denoted $n$, and $m$ stands for the number of source transitions, having no upstream place. If $t_{i}, t_{j} \in T$, the unique place relying $t_{j}$ to $t_{i}$ is denoted $p_{i j}$, if any, the corresponding delay is denoted $\tau_{i j}$ and the marking of this place is denoted $m_{i j}$. The maximal delay arising in the graph is denoted $\tau^{\max }$, i.e. $\tau^{\max }=\max _{p_{i j} \in P}\left\{\tau_{i j}\right\}$.

A path, denoted $\alpha$, from transition $t_{i}$ to transition $t_{j}$ is a sequence of transitions and places, of the form $\left(t_{i}, p_{i k_{1}}, t_{k_{1}}, p_{k_{1} k_{2}}, t_{k_{2}}, \ldots, t_{j}\right)$, where $p_{i k_{1}}, p_{k_{1} k_{2}}, \ldots \in P$. We denote $\tau_{\alpha}$ the sum of delays along path $\alpha, \tau_{\alpha}=\sum_{p_{k l} \in \alpha} \tau_{k l}$.

One associates to each transition of the considered timed event graph a function of timet, corresponding to the cumulated number of firings of the transition at time $t$. Such a function is called a counter. The counters corresponding to source transitions form the components of a vector, $u(t) \in \overline{\mathbb{R}}_{\text {min }}^{m}$, and the ones corresponding to the other transitions form the components of the vector, $\theta(t) \in \overline{\mathbb{R}}_{\text {min }}^{n}$.
As it is well known (see [4]), the dynamical behaviour of a timed event graph can be expressed by means of a linear equation over $\overline{\mathbb{R}}_{\text {min }}$, as follows.

$\theta(t)=\bigoplus_{\tau=0}^{\tau^{\max }}\left(A_{\tau} \cdot \theta(t-\tau) \oplus B_{\tau} \cdot u(t-\tau)\right)$,

where $A_{\tau} \in \overline{\mathbb{R}}_{\min }^{n \times n}$ is a matrix which entry $A_{\tau, i j}$ equals to $m_{i j}$, the number of initial tokens in place $p_{i j}$, if this place exists and the associated delay is $\tau$, and $\varepsilon$ else. Similarly, the entries of matrices $B_{\tau} \in \overline{\mathbb{R}}_{\text {min }}^{n \times m}$ correspond to the initial tokens of the places following source transitions.

Equation (1) is implicit in general. It is worth replacing it by the following explicit equation,

$\theta(t)=\underset{\tau>0}{\oplus}\left(A_{0}^{*} \cdot A_{\tau} \cdot \theta(t-\tau) \oplus A_{0}^{*} \cdot B_{\tau} \cdot u(t-\tau)\right)$,

where $A_{0}^{*}$ is the Kleene star of $A_{0}$, defined in the previous section.

\section{Explicit and state equation}

We shall now state some simplifying hypothesis, these are the following.

$\left(H_{1}\right)$ All the delays equal 0 or 1 . Analogously to the case of usual linear systems, the explicit equation 2 can be brought in state space form, if all the delays in the timed event graph are commensurable to a single delay. We can assume without loss of generality that this elementary delay equals 1 , this is the hypothesis $\left(H_{1}\right)$. For obtaining a state space model, we first expand all the places with delay $\tau>1$ into $\tau$ places with delays equal to 1 . Hence one adds $(\tau-1)$ intermediate transitions.

The added intermediate transitions are associated to counters that form the components of a vector, $\bar{\theta}(t) \in \overline{\mathbb{R}}_{\min }^{n^{\prime}}$, and we denote $x(t)$ the resulting extended state vector, $x(t)=\left[\begin{array}{l}\theta(t) \\ \bar{\theta}(t)\end{array}\right]$, which belongs to $\overline{\mathbb{R}}_{\text {min }}^{N}$, with $N=n+n^{\prime}$.

Further one assumes that:

$\left(H_{2}\right)$ The control acts without delay, i.e. all the delays associated to places with a source transition downstream are equal to 0 . This is not limiting, one can always add intermediate transitions and places so that the resulting extended graph satisfies this assumption. The dynamic of the expanded timed event graph is then described by an equation of the form $x(t)=\hat{A}_{0} \cdot x(t) \oplus \hat{A}_{1} \cdot x(t-1) \oplus \hat{B} \cdot u(t)$, which can be rewritten into the following explicit form, where $A=\hat{A}_{0}^{*} \cdot \hat{A}_{1}$ and $B=\hat{A}_{0}^{*} \cdot \hat{B}$, 
$x(t)=A \cdot x(t-1) \oplus B \cdot u(t)$.

All these notations permit to point out that the behaviour of a controlled timed event graph is deterministic, depending on the input $u(t)$ and on some initial conditions. This dependence can be explicited, and we shall use the following formulation:

$x(t)=A^{\tau} . x(t-\tau) \oplus\left[\bigoplus_{k=0}^{\tau-1} A^{k} . B . u(t-k)\right]$,

which holds true, for every $\tau \geq 1$.

In the following, we shall assume that the input $u(t)$ is actually a control, which can be arbitrarily assigned. For instance in a production process, the input can correspond to the authorization of performing a certain operation. Typically the beginning of a task performed by a robot, for instance, is subject to such a control input.

\section{PROBLEM FORMULATION}

\section{A. Temporal constraint}

Strict time constraints are frequent in industrial processes. One can for instance consider the example of a production process with a furnace for realizing a thermal treatment. The duration of any treatment in the furnace is fixed, or defined by a time interval. One wants to control the system to respect this constraint. The definition of a timed event graph already takes into account a delay on each place that corresponds to a minimal holding time. The maximal duration appears as an additional constraint that should be verified. Rather than a verification problem, we formulate the question as a control problem.

Hence $p_{i j}$ is the place subject to a strict constraint. An interval $\left[\tau_{i j}^{\min }, \tau_{i j}^{\max }\right]$ of time is associated to this place, where is a strict constraint, with $\tau_{i j}^{\min }=\tau_{i j}$.

This constraint is expressed through the following inequality, $m_{i j} x_{j}\left(t-\tau_{i j}\right) \geq x_{i}(t) \geq m_{i j} x_{j}\left(t-\tau_{i j}^{\max }\right)$,

where $m_{i j}$ is the initial marking of the place $p_{i j}$. The left

inequality is already taken into account by the linear model (3), so that the second one, say:

$x_{i}(t) \geq m_{i j} x_{j}\left(t-\tau_{i j}^{\max }\right)$,

where the product is over $\overline{\mathbb{R}}_{\text {min }}$, is actually the additional constraint to be validated.

\section{B. Causal feedback}

We consider a process modelled by (3), subject to the additional constraint (5). We want to determine a control $u(t)$ ensuring the respect of (5) for $t>0$. We shall a priori research this control in the form of a well posed causal feedback of the form, $u(t)=F . x(t-1)$, for $t>1$, where the product is in the sense of the Min-Plus algebra, and
$F \in \overline{\mathbb{R}}_{\text {min }}^{m \times N}$

Remark 1. A static control of the form $u(t)=F \cdot x(t)$ may result in an implicit loop, actually blocking the system. That is why we prefer using a delayed control law, always leading to a well-posed closed-loop system. Consider, for instance, the event graph of Fig. 1, and assume that this graph is subject to the additional constraint $x_{2}(t) \geq x_{1}(t-3)$. We remark from the graph equations that $u(t) \geq x_{1}(t)$, therefore one can choose $u(t)=x_{2}(t)$, trying to validate the constraint. This control law is of the form $u(t)=F . x(t)$, with $F=\left(\begin{array}{lll}\varepsilon & e & \varepsilon\end{array}\right)$. One can finally check that, actually, this is an implicit control law, resulting to a blocking of the closedloop controlled event graph.

\section{CONTROL SYNTHESIS}

\section{A. Single constraint and single control}

We propose in [3] a method for the synthesis of a control law solving our problem, provided that the following additional hypothesis is satisfied.

$\left(H_{3}\right)$ There exists a path $\alpha$ from $t_{u}$ to $t_{j}$, and we denote $\tau_{\alpha}$ the cumulated delay along this path.

Taking $\tau=\tau_{\alpha}$ in (4), and from the definition of $t_{u}$, we have $x_{j}(t) \leq\left(A^{\tau_{\alpha}}\right)_{j u} x_{u}\left(t-\tau_{\alpha}\right)$, and $x_{u}(t) \leq u(t)$, from which it is clear that:

$x_{j}(t) \leq\left(A^{\tau_{\alpha}}\right)_{j u} u\left(t-\tau_{\alpha}\right)$.

Applying again (4) with $\tau=\phi$, we obtain the following explicit expression:

$x_{i}(t)=\bigoplus_{r=1}^{N}\left(A^{\phi}\right)_{i r} x_{r}(t-\phi) \oplus\left[\bigoplus_{k=1}^{\phi-1}\left(A^{k} B\right)_{i} u(t-k)\right]$,

for every integer $\phi \geq 1$, which is the key to obtain the following result.

Theorem 1. Taking $\phi=\tau_{i j}^{\max }+\tau_{\alpha}+1$, the inequality,

$$
u(t) \leq \bigoplus_{r=1}^{N}\left(\left(A^{\phi}\right)_{i r}-\left(A^{\tau_{\alpha}}\right)_{j u}-m_{i j}\right) x_{r}(t-1)
$$

defines causal controls which guarantee that constraint (5) is satisfied if the two following sets of conditions hold :

(i) $\left(A^{\phi}\right)_{i r} \geq\left(A^{\tau_{\alpha}}\right)_{j u}+m_{i j} \quad$ for $r=1$ to $N$,

(ii) $\left(A^{k} B\right)_{i} \geq\left(A^{\tau_{\alpha}}\right)_{j u}+m_{i j} \quad$ for $k=0$ to $(\phi-1)$.

Proof. See [3].

Remark 2. The sufficient conditions depend on the initial markings of place $p_{i j}$ and of the path $\alpha$.

Corollary 1. There always exists a causal control validating constraint (5), if all initial markings of place $p_{i j}$ 
and of places of path $\alpha$ from $t_{u}$ to $t_{i}$ are null. Such a control is given by:

$$
u(t)=\bigoplus_{r=1}^{N}\left(A^{\phi}\right)_{i r} x_{r}(t-1) .
$$

Remark 3. It is important to notice that the control law proposed in theorem 1 is suboptimal. It is not optimal in general.

In our context, a control law $u^{\prime}(t)$ is said to be more permissive then a control $u(t)$ if it majorizes it in the sense of the usual order,

$$
u^{\prime}(t) \geq u(t), \forall t \geq 0 .
$$

Therefore, one could call optimal a control $u^{*}$ if the inequality

$$
u^{*}(t) \geq u(t), \forall t \geq 0
$$

holds for every control $u(t)$ guaranteeing the respect of the considered temporal constraint. The initial conditions $x(0)$ should be fixed to give sense to this definition. The problem is then that, even with a fixed initial condition, the existence of such an optimal control is not ensured. This simply comes from the fact, that, in general, if $x(t)$ and $x^{\prime}(t)$ are the solutions respectively corresponding to the controls $u(t)$ and $u^{\prime}(t)$, then $\max \left(x(t), x^{\prime}(t)\right)$ is not always the solution corresponding to the control $\max \left(u(t), u^{\prime}(t)\right)$. Then the existence of a most permissive control for the problem of time constraints is not guaranteed.

\section{B. Control synthesis to the case of multiple constraints}

We consider now the case of a timed event graph, having one source transition which is a control, but $Z$ places are constrained, noted $p_{z}$, for $z=1$ to $Z$. For each constrained place $p_{z}$, let $m_{z}, \tau_{z}$ and $\tau_{z}^{\max }$ respectively denote the initial marking, the minimal and maximal delays. Further, let $t_{z}$ and $t_{z}^{\prime}$ respectively denote the input and output transitions of the place, $x_{z}$ and $x_{z}^{\prime}$ denote the corresponding counters, and $\lambda_{z}$ denote the cumulated delay along a path going from $t_{u}$ to $t_{z}$. These constraints are expressed by the inequalities:

$m_{z} x_{z}\left(t-\tau_{z}\right) \geq x_{z}^{\prime}(t) \geq m_{z} x_{z}\left(t-\tau_{z}^{\max }\right)$,

for $z=1$ to $Z$.

We denote $u_{z}(t)$ the control law that satisfies the corresponding constraint (8).

Theorem 2. The equation, $u=\bigoplus_{z=1}^{Z} u_{z}(t)$, with

$$
u_{z}(t)=\bigoplus_{r=1}^{N}\left[\left(\left(A^{\phi_{z}}\right)_{x^{\prime} r}-\left(A^{\tau_{z}}\right)_{x u}-m_{z}\right) x_{r}(t-1)\right],
$$

and $\phi_{z}=\tau_{z}^{\max }+\tau_{z}+1$, for $z=1$ to $Z$, defines a causal control ensuring the respect of all constraints (8), if the two following sets of sufficient conditions are satisfied: (iii) $\left(A^{\phi_{z}}\right)_{x^{\prime} r} \geq\left(A^{\tau_{z}}\right)_{x u}+m_{z}$

(iv) $\left(A^{k} B\right)_{x^{\prime}} \geq\left(A^{\tau_{z}}\right)_{x u}+m_{z}$

for $r=1$ to $N$,

for $z=1$ to $Z$.

Proof. See [3].

Remark 4. The satisfaction of simultaneous constraints leads to the simultaneous satisfaction of the corresponding conditions, which is quite natural. In this sense, the extension of Theorem 1 that is done in Theorem 2 is not restrictive.

\section{Generalization to the case of multiple controls}

Now, we consider in this section a timed event graph with $m$ source transitions. Firstly, we suppose that $p_{i j}$ is the single place subjected to an additional temporal constraint. We calculate a vector, $u(t) \in \overline{\mathbb{R}}_{\text {min }}^{m}$, with $m \geq 1$, which is a control law that must satisfy the constraint (5). For each transition source, a variable counter is associated, noted $u_{s}(t)$, for $s=1$ to $m$. We also note by $\lambda_{s}$, the cumulated delay along a path going from $t_{u_{s}}$ to $t_{j}$.

Theorem 3. The respect of the temporal constraint (5), is guaranteed if:

(a) there exits $s$ such that:

$u_{s}(t) \leq \oplus_{r=1}^{N}\left[\left(\left(A^{\phi_{s}}\right)_{i r}-\left(A^{\lambda_{s}}\right)_{j u_{s}}-m_{i j}\right) x_{r}\left(t-\phi_{s}+\tau_{i j}^{\max }+\lambda_{s}\right)\right]$

with $\phi_{s}=\tau_{i j}^{\max }+\lambda_{s}+1, u_{l}(t)=\varepsilon$ for $l \neq s$, and

(b) the both following sets of conditions are satisfied:

(v) $\left(A^{\phi_{s}}\right)_{i r} \geq\left(A^{\lambda_{s}}\right)_{j u_{s}}+m_{i j} \quad$ for $r=1$ to $N$,

(vi) $\left(A^{k} B\right)_{i i^{\prime}} \geq\left(A^{\lambda_{s}}\right)_{j u_{s}}+m_{i j}$ for $k=0$ to $\left(\phi_{s}-1\right)$, and for $i^{\prime}=1$ to $m$.

Proof. We replace $\tau$ by $\lambda_{s}$ in (4), and from the definition of the function counter of $t_{j}$, we have,

$x_{j}(t) \leq\left(A^{\lambda_{s}}\right)_{j u_{s}} u_{s}\left(t-\lambda_{s}\right)$.

Applying again (4) for $\tau:=\phi_{s}$, we obtain, $\left.x(t)=A^{\phi_{s}} x\left(t-\phi_{s}\right) \oplus\left[\underset{\oplus_{k=0}^{\phi_{s}-1}}{\oplus} A^{k} B u(t-k)\right)\right]$, in particular,

$x_{i}(t)=\bigoplus_{r=1}^{N}\left(A^{\phi}\right)_{i r} x_{r}\left(t-\phi_{s}\right) \oplus\left[\bigoplus_{k=0}^{\phi_{s}-1}\left(\bigoplus_{i^{\prime}=1}^{m}\left(A^{k} B\right)_{i i^{\prime}} u_{i^{\prime}}(t-k)\right)\right]$.

Taking (10) into account, it appears that constraint (5) is satisfied if the both following conditions hold,

$\bigoplus_{r=1}^{N}\left(A^{\phi_{s}}\right)_{i r} x_{r}\left(t-\phi_{s}+\tau_{i j}^{\max }\right) \geq m_{i j} x_{j}(t)$, and,

$\phi_{s}-1 \quad m$

$\underset{k=0}{\phi_{s}-1}\left(\bigoplus_{i^{\prime}=1}^{m}\left(A^{k} B\right)_{i i} u_{i^{\prime}}\left(t-k+\tau_{i j}^{\max }\right)\right) \geq m_{i j} x_{j}(t)$.

Father, taking (9) into account, these conditions become $\bigoplus_{r=1}^{N}\left(\left(A^{\phi_{s}}\right)_{i r}-m_{i j}\right) x_{r}\left(t-\phi_{s}+\tau_{i j}^{\max }\right) \geq\left(A^{\lambda_{s}}\right)_{j u_{s}} u_{s}\left(t-\lambda_{s}\right)$, and, 
$\underset{k=0}{\phi_{s}-1}\left[\bigoplus_{i^{\prime}=1}^{m}\left(\left(A^{k} B\right)_{i i^{\prime}}-m_{i j}\right) u_{i^{\prime}}\left(t-k+\tau_{i j}^{\max }\right)\right] \geq\left(A^{\lambda_{s}}\right)_{j u_{s}} u_{s}\left(t-\lambda_{s}\right)$.

Choosing, $\phi_{s}=\tau_{i j}^{\max }+\lambda_{s}+1$, conditions (v) and (vi) being verified, and the control law satisfying the inequality of the theorem, one can check that the constraint (5) is satisfied.

Corollary 2. Let a timed event graph with $m$ source transitions $(m \geq 1)$ and $\mathrm{Z}$ additional temporal constraints of type (8) $(Z \geq 1)$. The causal control law which guarantees the respect of the $Z$ constraints (8) is defined by:

For $l=z$ to $Z$, $u_{l}(t)=\bigoplus_{z=1}^{Z} u_{z}(t)$,

where $u_{z}(t)$ is the control law, calculated by Theorem 3, to check the $\mathrm{z}^{\text {th }}$ constraint and $u_{l}(t)=\varepsilon$ for $l \neq z$.

Proof. A control law $u_{z}(t)$, validates the $\mathrm{z}^{\text {th }}$ constraint, if conditions (v) and (vi) of Theorem 3 are satisfied. Thus, we have, for $z=1$ to $Z, u_{z}(t) \geq \underset{z=1}{Z} u_{z}(t)$. According to Theorem 3 , it is clear that the control law $u_{l}(t)=\oplus_{z=1}^{Z} u_{z}(t)$ for $l=z$ to $Z$, guarantees the respect of all $Z$ temporal constraints.

\section{EXAMPLE}

Consider the timed event graph of Fig.1. This graph contains two source transitions modelling respectively, control $u_{1}$ and control $u_{2},(m=2)$.

Two additional temporal constraints, $Z_{1}$ and $Z_{2}$, are added to this graph, and are expressed respectively by the following inequalities: $x_{2}(t) \geq x_{1}(t-1), x_{3}(t) \geq x_{2}(t-1)$, for $t \geq 1$.

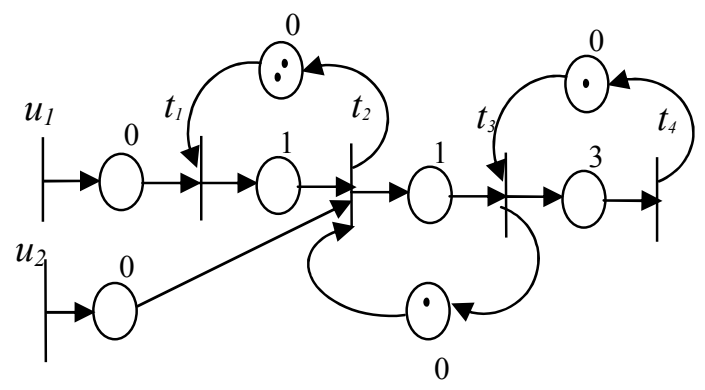

Fig. 1. Timed event graph-Example

The problem consists in calculating a control vector, $u(t)=\left(\begin{array}{l}u_{1}(t) \\ u_{2}(t)\end{array}\right)$ which satisfies these both constraints.

By applying Corollary 2, the previous graph, see Fig. 1, has been transformed into that of Fig. 2, with $\tau^{\max }=1$. To do so, place $p_{43}$ has been split into three places timed to 1 . The state equations associated with this new timed event graph, see Fig. 2, is:

$x(t)=\left(\begin{array}{llllll}2 & 3 & \varepsilon & \varepsilon & \varepsilon & 4 \\ e & 1 & \varepsilon & \varepsilon & \varepsilon & 2 \\ \varepsilon & e & \varepsilon & \varepsilon & \varepsilon & 1 \\ \varepsilon & \varepsilon & \varepsilon & \varepsilon & \varepsilon & e \\ \varepsilon & \varepsilon & e & \varepsilon & \varepsilon & \varepsilon \\ \varepsilon & \varepsilon & \varepsilon & \varepsilon & e & \varepsilon\end{array}\right) x(t-1) \oplus\left(\begin{array}{ll}e & \varepsilon \\ \varepsilon & e \\ \varepsilon & \varepsilon \\ \varepsilon & \varepsilon \\ \varepsilon & \varepsilon \\ \varepsilon & \varepsilon\end{array}\right) u(t)$,

where the components of $x(t)$ are the functions of counters associated to transitions $t_{1}, t_{2}, t_{3}, t_{4}, t_{5}$ and $t_{6}$, and the vector $u(t)$ is the control law. We shall then apply Corollary 2 to calculate a control $u(t)$ which guarantees the respect of the both temporal constraints $Z_{1}$ and $Z_{2}$.

For this example, it is enough to find, for each temporal constraint, only one component of the vector $u(t)$ to guarantee the respect of this constraint. Firstly, we determine a component of the vector $u(t)$, which satisfies constraint $Z_{1}$. For this example, we have, $\tau_{i j}^{\max }=\tau_{21}^{\max }=1$, and $\lambda_{1}=e$, the delay of the path $\alpha_{1}$ from transition $u_{1}$ to a transition $t_{1}$. The initial marking of the place $p_{21}$ is $m_{i j}=m_{21}=e$, and the initial marking of the path $\alpha_{1}$ is $\left(A^{\lambda u_{1}}\right)_{j u_{1}}=\left(A^{e}\right)_{1 u_{1}}=e$. We choose,

$\phi_{1}=\tau_{21}^{\max }+\lambda_{1}+1:=2$.

We take account of (4) with $\tau=2$, the state equation this example is equivalent to the following expression
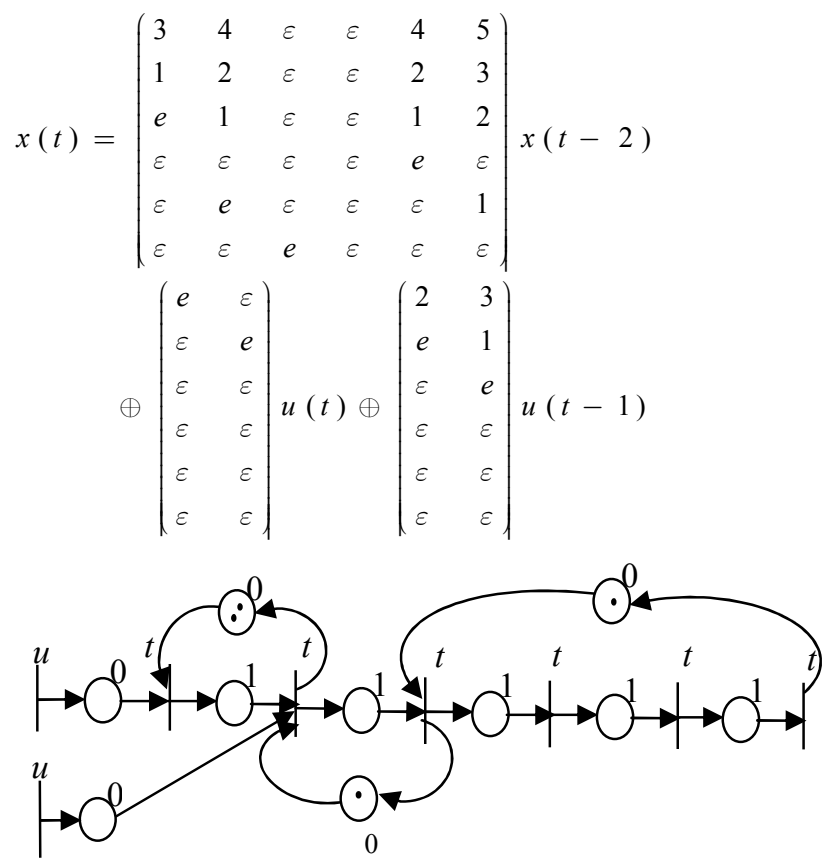

Fig. 2. Extended timed event graph

According to Corollary 1, conditions (v) and (vi) of Theorem 
3 are checked. Thus, the equation which guarantees the respect of constraint $Z_{1}$ is,

$$
\begin{aligned}
u_{1}(t) & =\bigoplus_{r=1}^{6}\left(A^{2}\right)_{2 r} x_{r}(t-1) \\
& =1 x_{1}(t-1) \oplus 2 x_{2}(t-1) \oplus 2 x_{5}(t-1) \oplus 3 x_{6}(t-1)
\end{aligned}
$$

Secondly, in a similar way, we calculate a control which satisfies constraint $Z_{2}$. In this case, we have, $\tau_{i j}^{\max }=\tau_{32}^{\max }=1$, and $\lambda_{2}=e$, which is the delay of path $\alpha_{2}$ from transition $u_{2}$ to $t_{2}$. The initial marking of place $p_{32}$ is $m_{i j}=m_{32}=e$, and the initial marking of path $\alpha_{2}$ is $\left(A^{\lambda_{2}}\right)_{j u_{2}}=\left(A^{e}\right)_{2 u_{2}}=e$. We choose, $\phi_{2}=\tau_{32}^{\max }+\lambda_{2}+1:=2$. According to Corollary 1 , the equation,

$$
\begin{aligned}
u_{2}(t) & =\stackrel{6}{\oplus}\left(A^{2}\right)_{3 r} x_{r}(t-1) \\
& =x_{1}(t-1) \oplus 1 x_{2}(t-1) \oplus 1 x_{5}(t-1) \oplus 2 x_{6}(t-1),
\end{aligned}
$$

is a component of a vector, $u(t)$, that assures the respect of constraint $Z_{2}$.

After the corollary 2, the control law which guarantees the respect of both temporal constraints, $Z_{1}$ and $Z_{2}$, is given by:

$u(t)=\left(\begin{array}{l}x_{1}(t-1) \oplus 1 x_{2}(t-1) \oplus 1 x_{5}(t-1) \oplus 2 x_{6}(t-1) \\ x_{1}(t-1) \oplus 1 x_{2}(t-1) \oplus 1 x_{5}(t-1) \oplus 2 x_{6}(t-1)\end{array}\right)$.

\section{CONCLUSIONS}

We have recalled two conditions which are sufficient for the existence of a causal control ensuring the satisfaction of a given temporal constraint in a controlled timed event graph. We have also applied this approach to the case of a timed event graph subject to multiple constraints. A generalization of this approach for timed event graphs with multivariable control has been proposed in this paper. This method is illustrated on an example. We trust that it could be valuable as well in different contexts, notably for the verification and validation of automated systems as well as telecommunication processes and real-time software.

\section{REFERENCES}

[1] Alur, R. and D. Dill, A Theory of Timed Automata, Theoretical Computer Science (TCS), Vol. 126, No. 2, pp. 183-235, 1994.

[2] Amari S., I. Demongodin and J.J. Loiseau, Sizing, cycle time and plant control using dioid algebra, Chapter 6 in Supply Chain Optimisation, Series Applied Optimization, A. Dolgui J. Soldek and O. Zaikin (Eds.), Springer. pp. 71-85, 2004.

[3] Amari S., J.J. Loiseau, and I. Demongodin, Control of Temporal Constraints Based on Dioid Algebra for Timed Event Graphs, 19th IEEE International Parallel \& Distributed Processing Symposium (IPDPS), Colorado, 2005.

[4] Baccelli F., G. Cohen, G.-J. Olsder, and J.-P. Quadrat, Synchronization and Linearity: An algebra for Discrete Event Systems, Willey, 1992.

[5] Berthomieu B. and M. Diaz, Modelling and verification of time dependant systems using time Petri nets, IEEE Transactions on Software Engineering, Vol. 17, No. 3, pp. 259-273, 1991.

[6] Bonhomme P., P Aygalin and S. Calvez, Systèmes à contraintes de temps : Validation, Evaluation et contrôle, $M S R$, Toulouse, 2001.

[7] Cofer D.D., V.K. Garg, Control of event separation times in discrete event systems. In Proceedings 34th IEEE Conference on Decision and Control, pp. 2005 - 2010, New Orleans, 1995.

[8] Ghezzi C., D. Mandrioli, S. Morasca and M. Pezze, A unified highlevel Petri net formalism for time critical systems, IEEE Transactions on Software Engineering, Vol. 17, No. 2, pp. 160-172, 1991.

[9] Holloway L.E., B.H. Krogh and A. Giua, A survey of Petri net methods for controlled discrete event systems, pp. 151-190, Kluwer Academic Publishers, Boston, 1997.

[10] Lahaye S., B. Cottenceau and A. Correïa, Commande des graphes d'événements temporisés avec contraintes de temps critique, In Proceedings Conf. Internationale Francophone d'Automatique (CIFA'04), Douz, Tunisie, 2004.

[11]Moody J., K. Yamalidou, M. Lemmon and P. Antsaklis, Feedbackcontrol of Petri nets based on place invariants, In Proceedings 33th IEEE Conference on Decision and Control, pp. 3104-3109, Florida, 1994. 